Running Head: DATA CHECKOUT FOR CONFIRMATORY SECONDARY ANALYSIS

NOTE: This version was uploaded on $7 / 30 / 18$ and has not yet undergone peer review; comments are very welcome! Please cite this paper as:

Scott, K. \& Kline, M. (2018). Preprint: Enabling Confirmatory Secondary Data Analysis by Logging Data 'Checkout'. Available at www.psyarxiv.com/87wjc, Accessed $<$ Date of Access $>$. DOI:10.31234/osf.io/87wjc

Enabling Confirmatory Secondary Data Analysis by Logging Data 'Checkout'

Kimberly M. Scott and Melissa Kline

Massachusetts Institute of Technology

Correspondence concerning this article should be addressed to Kimberly Scott, 46-4009, 77

Massachusetts Ave, Cambridge, MA 02139.Email: kimscott@mit.edu 


\begin{abstract}
As more researchers make their datasets openly available, the potential of secondary data analysis to address new questions increases. However, the distinction between primary and secondary data analysis is unnecessarily confounded with the distinction between confirmatory and exploratory research. We propose a framework, akin to library book checkout records, for logging access to datasets in order to support confirmatory analysis where appropriate. This would support a standard form of preregistration for secondary data analysis, allowing authors to demonstrate that their plans were registered prior to data access. We discuss the critical elements of such a system, its strengths and limitations, and potential extensions.
\end{abstract}




\section{Enabling Confirmatory Secondary Data Analysis by Logging Data 'Checkout'}

Many scientific practices are based on norms rather than strict rules from our institutions, professional societies, or funders. These norms evolve over time - for example, methods sections of psychology papers have changed greatly since the 1950 s, thanks to advances in graphing methods, the reduced role of page limits in paper journals, and changes in the scientific community's beliefs about what information is appropriate or necessary to share. In the last five years, norms around how we replicate and share data have been changing rapidly, to promote both increased transparency in our processes and an increased focus on ensuring - and testing the robustness of individual claims (Nosek \& Lindsay, 2018). These changes are supported by changing technologies and practices, from general tools like shareable online documents to purpose-built tools including platforms like the Open Science Framework (Center for Open Science, 2018), which lower the workload for individual scientists looking to implement these practices.

Preregistration of experimental studies is a newly developing norm in psychology that allows researchers to clearly mark the distinction between decisions made before and after viewing their data, and correspondingly the difference between their confirmatory and exploratory analyses. While the majority of experimental papers in psychology journals are written as though all hypotheses were made and analyses planned before data collection (Wagenmakers, Wetzels, Borsboom, van der Maas, \& Kievit, 2012), we now know that this is not always the case, thanks to analytical tools such as p-curve and increased transparency by researchers (Simonsohn, Nelson, \& Simmons, 2014; John, Loewenstein, \& Prelec, 2012). On mathematical grounds, the results of pre-specified analysis constitute stronger evidence of a 
claim than same results of the same analysis designed based on initial viewing of the data, even when the researcher does not actually try multiple approaches (Gelman \& Loken, 2013) and even when using Bayesian methods (Dienes, 2016), due to the vast number of methodological and analytical decisions available (Wicherts, Veldkamp, Augusteijn, Bakker, van Aert, \& van Assen, 2016).

Currently, however, the distinction between exploratory and confirmatory research is confounded by the distinction between the use of new and existing datasets. It is generally more complicated, and in some cases prohibited, to preregister analysis of existing data using standard templates (e.g. https://aspredicted.org). Preregistering only when preparing to collect new data limits the strength of the conclusions we can draw when reconsidering original analysis or when addressing new questions with existing datasets. Consider the case of Meltzoff et al. (2016), who set out to explain why Oostenbroek et al. (2016) failed to replicate an earlier finding of neonatal imitation of facial expressions (Meltzoff \& Moore, 1977). Their rebuttal outlines 11 ways in which Oostenbroek et al.'s design reduced the probability of detecting an effect, and their own re-analysis shows that infants were consistently significantly above chance on one of the behaviors tested (tongue protrusion). Yet despite plausible concerns, careful analysis, and the cooperation between the two groups in sharing data, the rebuttal is necessarily weakened by the knowledge that a dataset can, with sufficient effort, generally be interpreted to support either preferred outcome. In this case, neither contribution achieved its full potential to finally resolve a debate about neonatal imitation: Oostenbroek et al.'s data got "one shot" to be used in confirmatory research, and Meltzoff et al.'s secondary analysis must be treated as exploratory even if it could have been specified prior to accessing the data. This is a particularly unfortunate outcome for neonatal imitation data, which is exceptionally difficult to collect (it often involves 
researchers camping out in maternity wards, hoping to catch a rare calm moment when a newborn is awake but neither crying nor eating). Similarly, very large datasets that have the potential to answer many questions from many researchers are often beyond the abilities of any one lab to collect - consider for instance the Health and Retirement Study (Juster \& Suzman, 1995), cited in over 3000 publications (University of Michigan, 2018), or the new NIH initiative "All of Us" (Collins \& Varmus, 2015), which aims to collect detailed longitudinal health data from over 1 million Americans. While these datasets are still exceptionally valuable for exploratory work, in principle a researcher who has not yet seen the data should also have the option to use them for confirmatory work.

The ability to reuse data to answer new questions is indeed one of the chief virtues of openly sharing data. While the use of existing datasets has a long history in many disciplines, the tradition in our own field of cognitive development has been independent collection of small datasets. Even here, the value of secondary dataset analysis is becoming increasingly clear (Davis-Kean \& Jager, 2017). Psychologists are already beginning to consider how to bring the same rigor and planning that preregistration provides to secondary data analysis, and norms for secondary data preregistration are emerging (Weston, Ritchie, Rohrer, \& Przybylski, 2018). When collecting experimental data, the distinction between decisions made before and after viewing the data is built in: a researcher can make decisions before the data even exist. But when data already exist, it is both harder to see the line and harder to convey to others what steps have been taken to limit analytic flexibility, which has led to doubt about the value of preregistration for secondary analyses (van 't Veer \& Giner-Sorolla, 2016; Watt \& Kennedy, 2017). Two kinds of solutions have been proposed: being as transparent as possible about the work conducted and whether knowledge of the data was used to plan analysis, and pledging to follow a set of 'best 
practices' in reusing a dataset responsibly (Heng, Wagner, Barnes, \& Guarana, 2018; Nosek, Ebersole, DeHaven, \& Mellor, 2018; DeHaven \& Mellor, 2018).

To complement and support these practices, we propose a small change in how we as a scientific community access openly shared data for secondary analysis: maintaining a central electronic "sign out sheet" for datasets, so that researchers can clearly and verifiably indicate at what point in the research process they accessed the data. We refer to the proposed practice as 'checking out' a dataset, analogous to checking out a book at a library. Most libraries allow anyone to check out books and resources, but not walk directly out the door with them - you have to check them out first, and the library retains a record of who has checked out which books. Critically, data checkout does not change who is authorized to access the dataset; it only introduces friction into the access process. In addition to supporting planned confirmatory analysis, data checkout would encourage all researchers to be more aware of the transition from pre- to post-data-access decision-making, allowing for more deliberate and open exploratory or confirmatory work. We anticipate that the proposed functionality would complement existing tools, promote good practices, and enhance the value of shared data.

\section{Implementation}

Throughout, we will refer to a researcher who collected and published a dataset as the 'dataset creator' (or 'creator') and one conducting secondary analysis as the 'dataset user' (or 'user').

Data checkout will be most seamlessly integrated into the research experience if it can be supported by the central resources already used for sharing data, such as journals' supplementary data repositories and the Open Science Framework. In many cases, almost all of the necessary functionality has already been implemented - for instance, journals may track file downloads for 
impact measurement purposes. The only change users would experience is that downloading data would require a login: for instance, clicking on a download link or opening files containing raw data might redirect to a 'checkout' page with a brief explanation of the reasons to track data access. However, upon login, data access would still be immediate. A user who wished to conduct confirmatory research on the existing dataset could therefore preregister their planned analyses before checking out the data, and simply include a statement like this in the preregistration:

The following is a list of key researchers on the project. As of this preregistration, we certify that the following people have not accessed the dataset [Title] at [link]:

[Names and links to individual checkout histories of key researchers]

After depositing the preregistration on a server like AsPredicted or the Open Science Framework, the researchers would then proceed to check out the data and conduct their analyses.

We describe below the four minimal technical elements of a data checkout system:

\section{Authentication system that identifies unique users}

The key claim a checkout system needs to verify is the following: None of the downloads of this data prior to [date] were by [researcher's user ID]. The more confident we are that each researcher has at most one user ID - essentially, the further from anonymous the login system - the more compelling this statement is. Leveraging existing tools such as institutional ORCID authentication (Haak, Fenner, Paglione, Pentz, \& Ratner, 2012) would allow a reasonable degree of confidence in the uniqueness of users without imposing burdensome demands on researchers. This must be balanced, however, against the need to allow access even to those without institutional affiliation.

\section{Central record of who downloaded what when}


This is the core of the data checkout proposal: whenever someone accesses the data, that access should be recorded. This is what allows a researcher to back up a claim of confirmatory research with a record that he or she did not access the dataset prior to designing the analysis.

Access to the full dataset must require authentication, so that the system providing access can record a timestamp and user each time the dataset is downloaded. If the dataset user chooses to make his or her checkout records for this dataset available, the system must then be able to, at minimum, verify the earliest time at which he or she accessed the dataset. Although the simplest implementation would simply be a public record of all downloads, note that verification of a particular data user's history does not necessarily entail publishing the identities of other researchers who have accessed the data.

The flexibility of potential reuse would be optimized by allowing researchers to specify a subset of the data to download - for instance, by experiment or dependent measure - to allow for cases where the answer to one question informs the design of the next. For ease of implementation, this could initially be approximated by allowing researchers sharing a dataset to split it into pieces that could be downloaded separately.

\section{Publicly accessible summary statistics for protected datasets}

In order to understand the potential value of the dataset and make informed decisions about analytic techniques, researchers must be able to freely access a summary of the available data. That is, prior to accessing the full dataset they should know the number of subjects, which dependent measures were collected on what scales, and so on. At minimum, this simply corresponds to the information that would be included in a 
methods section. Where possible, a jittered dataset (one that has the same structure, but randomly scrambled values) may be provided to allow researchers to design, test, and register the exact code they wish to use for analysis.

\section{Simple license for protected data that encourages central access}

To maintain a complete record of individuals who have accessed a dataset, the system described must be the sole means of public access to the data (or coordinate with any other systems providing access to share records - for instance, a journal and an open repository could share a record for a particular resource stored in both locations). In downloading the dataset, researchers should by default accept a simple license that discourages publishing the raw data elsewhere. This is, fortunately, already a widely-held social norm in the field when conducting secondary analyses: retaining the connection to the original dataset is important for properly crediting the original authors.

Logging data checkout is a small but potentially powerful step that would neatly complement the emerging network of tools for sharing data and for linking data, projects, and researchers. We expect it will be especially valuable for datasets that are difficult to obtain (e.g., developmental research, special populations, longitudinal studies) and for rich datasets that may shed light on multiple questions. In cases where a researcher wishes to challenge the conclusions of a study, or where the data is so precious that it will be impossible or very costly to get new datasets, being able to establish evidence of this delay (with a preregistration and subsequently an access timestamp) allows stronger conclusions, sharper critiques, and better use of our existing datasets to make scientific progress.

\section{Data checkout in practice: questions and challenges}


Technology alone cannot ensure responsible reuse of data, nor will checkout be appropriate in all cases. As with most new practices in sciences, data checkout solves some problems in some situations. While it is not a panacea, we believe that data checkout can add considerable value to the strength of the inferences we make when re-analyzing datasets, at relatively low time cost. Understanding the strengths and weaknesses of this tool is critical for its success.

\section{Won't data checkout discourage exploratory work?}

Data checkout is a tool to complement preregistering planned analyses; it does not directly affect the exploratory workflow. However, many researchers (e.g., GoldinMeadow, 2016; Scott, 2013) harbor concerns that moving norms in psychology toward explicitly confirmatory work will hamper creativity and prevent the serendipitous discovery of patterns that can launch new questions and theories (Washburn et al., 2018). We anticipate two primary concerns about data checkout as regards exploratory research:

First, data checkout could serve to "out" exploratory work as such. We believe that better recognition of the distinction would in fact strengthen the position of exploratory research by making this a neutral revelation. Exploratory research plays a vital and unique role in the scientific process (Whewell, 1858) but is currently hobbled by a strong expectation that nearly all studies will be presented as confirmatory (whether concerning new or reused datasets). In this model, exploratory research - which allows researchers to fully explore and identify potentially unexpected trends in a dataset - ends up unfairly painted as subpar or misleading confirmatory work. By proudly recognizing carefully conducted, exciting exploratory work as such, we believe we would raise its status, avoiding an inappropriate dominance of confirmatory research. We might realize 
that many of the studies we would hold up as "role models" of confirmatory research are in fact exploratory, both removing stigma for exploratory work and reducing the unrealistic expectation for confirmatory work to regularly discover surprising new phenomena.

Second, data checkout might lead researchers to delay exploratory work for fear of missing out on the opportunity for later confirmatory work. Indeed, encouraging thoughtfulness about accessing data is intended to encourage researchers to step back and consider their options, much like your computer's operating system provides an extra warning before irreversible actions. We believe that adding this small amount of friction will only support researchers in acting more consistently with their genuine preferences. If they choose not to access a dataset immediately upon being reminded that this means their work is exploratory - but would otherwise have forged ahead - we can reasonably assume that their intent was in fact to do confirmatory research or that they were undecided, and that the reminder was in their best interest. As we all get better at distinguishing between exploratory and confirmatory work and deciding which approach is most appropriate for a particular question, and as the contributions of exploratory work are better recognized, researchers will be more comfortable deciding when to access a dataset and forgo the opportunity to do truly confirmatory work.

\section{Does data checkout mean that I have to preregister all of my secondary data analysis now?}

No. As with other proposed reforms, data checkout is simply the addition of a tool. It serves to increase the weight of a secondary data analysis preregistration if the researcher wishes to create one. It will remain possible to conduct exploratory research 
with secondary datasets, and to conduct confirmatory research simply by refraining from exploring the data first, without a formal preregistration.

\section{What CAN'T data checkout fix?}

Data checkout serves a specific function as a way to enable confirmatory testing of hypotheses using existing datasets. However, it does not eliminate the necessity of a basic degree of trust. For instance, data checkout cannot prevent outright cheating: just as a person could falsify a dataset, they could give false information on a preregistration (e.g. altering data to appear that it was collected after, not before, the preregistration was submitted), or falsely log their data checkout after getting the data from a colleague or making an alternate login account. Science indeed depends on being able to trust that our colleagues are not deliberately lying - data checkout does not change this.

Beyond this, many of our scientific practices will remain just as necessary, and new tools will be required to solve other aspects of the challenges with robustness and replicability we currently face. For instance, data checkout cannot prevent the problem of selective attention to controversial or surprising datasets. Just as publication bias can prevent 'boring' null results from being published, our own biases mean that we are more likely to turn our powerful microscopes toward datasets we find surprising or questionable; we are unlikely to pour our efforts into minutely checking the analytic soundness of a dataset that produces a more prosaic result.

Finally, data checkout doesn't prevent another scientist from using a dataset to reach a conclusion that its creators dislike: it is a tool to make users more mindful about making the leap to access a dataset, not to allow the creator to gate-keep according to the hypotheses a user wants to test. However, existing restrictions on who may access a 
dataset (for instance, if IRB certification is required) are compatible with an additional checkout layer.

\section{In which situations would data checkout would be inappropriate?}

We have described the positive function of data checkout above, but there may be cases where data checkout will either be unhelpful or could be actively harmful. First, if the dataset is very well known in a field - widely analyzed, already available and circulated among many scientists (as with some corpora of child and child-directed speech, e.g. Brown, 1973) - data checkout could create a false sense of rigor. Second, if there is some reason that scientists (or citizens more generally) could be targeted for having looked at a particular dataset, leaving a dataset unlogged may be appropriate. This includes cases where accessing a dataset could be construed as revealing sensitive information about the user's beliefs or group membership, or where a checkout record could compromise their relationship with an employer or eligibility for a position. We expect that these cases, while limited, can be addressed using similar principles to those used when deciding whether to share human subjects data.

Another reason researchers might choose to not employ checkout is that a dataset has special potential for exploratory research or scientific outreach: where even asking people to log in to view the data would impose an unacceptable barrier. One example is WordBank (Frank, Braginsky, Yurovsky, \& Marchman, 2017), a dataset of crosslinguistic vocabulary development inventory questionnaires presented via an interactive website. These data are of interest both to researchers and to parents and other members of the public. A scientist wishing to use a resource like this for confirmatory research would need to proceed as we currently proceed with preregistrations on existing data: by 
being explicit about what was accessed when and what they already know about the dataset.

\section{Won't this make fewer people access my data? I want credit for my open datasets!}

Data checkout is indeed designed to interpose 'friction' in between another researcher and your data, to encourage them to distinguish between confirmatory and exploratory work. On the other hand, a checkout record both enhances the value of the data you have created by giving it more power to answer additional questions, and lets you track the impact of your dataset in a way that can be useful for your own scientific career.

\section{How can I specify my (confirmatory) analyses in enough detail if I can't access the data first?}

Many scientists (including ourselves!) are used to "getting our hands dirty" with a dataset as quickly as possible--learning about the dataset while we clean or summarize it, make initial graphs, and work our way through understanding the variables. This approach is both eminently practical and a danger: regardless of the researcher's intent, it provides impressionistic insights into the data that muddy the waters of any confirmatory tests. Those who share their datasets can make life easier for other researchers by providing clear summary information about the dataset - how it was collected, what variables it contains, and ideally a full data dictionary (Broman \& Woo, 2018) - so that analyses can be planned without first looking at the data. Publishing any code already used to analyze the data, in addition to supporting transparency and computational replicability, also serves as a helpful concrete example to other users. In some cases, 
dataset creators may choose to provide a 'jittered' version of a dataset that preserves its structure while scrambling the content; this can allow users to make more specific predictions during pre-registration and even implement code for carrying out specific analyses. As with many open-science tools, norms, and practices, the steps we take to make our science more usable and robust tend to be applicable outside their originally envisioned context: data dictionaries are helpful for any reuse of a dataset, whether or not the researcher is additionally able to access the raw data when planning analyses.

\section{What if lots of other people have already looked at this dataset? Won't datasets get 'overused'?}

Over time, datasets will become more fully characterized and more subject to existing biases. Data checkout will not solve this problem, but will make it easier to identify. We won't be able to register that a user has read another paper about the dataset and therefore has some (or possibly a lot of) knowledge about it, although with clear links between datasets and results we may be able to see what was published about the dataset at the time of registration. Researchers will still need to be transparent about what knowledge they have about a dataset at the time they preregister. But we will at least be able to distinguish between a dataset that's been checked out 10,000 times and one that's been checked out four times.

\section{It's already possible to make an analysis plan before viewing the data - why bother tracking access? Can't we trust each other?}

In many cases, researchers are already making principled and transparent decisions about when to access data, but don't receive the credit they should, because there is no standard way to convey this rigor. While science is in many ways a trust-based 
enterprise, adopting preregistration amounts to acknowledgement that evidence of compliance provides additional support for a claim, just as open data and materials do. Likewise, a standard method to document when an existing dataset was first accessed by a given researcher, coupled with preregistration before that date, could serve the same function.

\section{My favorite [repository/supplemental journal archive] doesn't support this!}

Please lobby your platforms to support data checkout! An 'honor code' system can be implemented by a researcher as a first step with very low engineering cost, for instance with a note asking anyone accessing the data to fill out a webform. In cases where data are *not* openly shared, the process used to share data (e.g., via personally emailing the authors) can serve as proof of first access date. But in many cases, data access is already being logged to track impact, and a full-fledged implementation would be straightforward. As with preregistration and paper formatting standards, journals are unlikely to converge on identical systems, but interoperability is an important goal, and developing norms and expectations is the first step to changing practices.

\section{Beyond basic data checkout}

A basic system for data checkout would form a foundation for a variety of additional functionality that could benefit researchers. For instance, extensions could:

- Formally link data checkout records, preregistrations, and results to make research more replicable and cumulative, and to give due credit to dataset creators.

- Allow a dataset user to check out an arbitrary subset of the data (and record that access), for instance to address a question that only pertains to a particular task or to document a cross- 
validation approach (where exploratory work on one subset of the dataset is tested on another).

- Provide an API that allows users to submit code to be run on a dataset and receive the results, without receiving the data itself, with central logging of all analyses conducted. This could provide even stronger evidence that dataset users did not conduct additional unreported analyses, make it easier to tell what questions have already been addressed, and protect participant privacy where necessary.

- Collect information about how people are using a dataset, allowing dataset creators to more easily track the scientific impact of their work.

- Encourage checkout of stimuli as well as data in cases where the exact details of stimuli may support alternative explanations for phenomena. Such post-hoc explanations are similar to post-hoc analyses; with sufficient effort it is often possible to find some aspect of the stimuli that could explain away a result. While such exploratory work is valuable and can suggest future controls, it is qualitatively different to propose the presence of a particular confound without having seen the stimuli, especially in the case of a failed replication.

- Implement data checkout for currently "closed" datasets where access is obtained by emailing the authors and hoping for a response: if such requests could be made and fulfilled via the journal website, for instance, then that communication would serve as documentation of first access, even without the data being hosted centrally. (As a side benefit, a record of responses could encourage authors to honor statements that data is available "upon request"!)

\section{Conclusion}

The challenge of preregistering secondary analyses arises from a welcome trend: scientists choosing to work openly and make their data available to the scientific community. 
'Closed data' provides a built-in solution to the problem of preregistering on existing datasets: when researchers have to personally request data or travel to access a dataset stored in a particular location (common in fields like economics and education that deal with datasets featuring significant personally identifying information), there is significant natural 'friction' that highlights the differences between hypothesizing with and without the data in hand, and often specific documentation (such as an email exchange) granting access to the data. This is just one example of how our practices will need to shift in response as our data becomes more open. As the amount of data we share grows, we will continue to face challenges in how best to document it, make it accessible to the community, and capitalize on its scientific potential. Just as the format and content of journal articles varies across contexts and studies while maintaining a basic form, we expect that how we share and access datasets will draw on a set of shared practices that provide clarity and scientific rigor.

The field of psychology is currently evolving toward a new platonic ideal of research output, stretching beyond the research article to include preregistration for confirmatory work, open materials and data, reproducible analyses, explicit statements on the limits of generalizability, and much more. The norms we create and adopt around this ideal will have the potential to revolutionize the reproducibility and rigor of our science.

\section{Disclosures}

Conflicts of interest: The authors declare that they have no conflicts of interest with respect to the authorship or the publication of this article.

Author contributions: The authors generated the idea for the article and drafted the manuscript together. Both authors approved the final submitted version of the manuscript.

Acknowledgements: We thank the SIPS (Society for the Improvement of Psychological Science) community. 


\section{References}

Broman, K. W., \& Woo, K. H. (2018). Data Organization in Spreadsheets. American Statistician, 72(1), 2-10. https://doi.org/10.1080/00031305.2017.1375989

Brown, R. (1973). A first language: The early stages. Harvard U. Press.

Center for Open Science (2018). The Open Science Framework. Accessed 2018-07-11 at http://www.osf.io

Collins, F. S., \& Varmus, H. (2015). A new initiative on precision medicine. N Engl J Med, 372(9), 793-795. https://doi.org/10.1056/NEJMp1500523

Davis-Kean, P. E., Jager, J., \& Maslowsky, J. (2015). Answering Developmental Questions Using Secondary Data. Child Development Perspectives, 9(4), 256-261. https://doi.org/10.1111/cdep.12151

DeHaven, A., \& Mellor, D. (2018). One preregistration to rule them all? Retrieved from https://cos.io/blog/one-preregistration-rule-them-all/

Dienes, Z. (2016). How Bayes factors change scientific practice. Journal of Mathematical Psychology, 72, 78-89. https://doi.org/10.1016/j.jmp.2015.10.003

Fenson, L., Marchman, V. A., Thal, D. J., Dale, P. S., \& Reznick, J. S. (2007). MacArthur-Bates communicative development inventories: User's guide and technical manual. Baltimore, MD: Brookes.

Frank, M. C., Braginsky, M., Yurovsky, D., \& Marchman, V. A. (2017). Wordbank: An open repository for developmental vocabulary data. Journal of Child Language, 44(3), 677694. https://doi.org/10.1017/S0305000916000209

Gelman, A., \& Loken, E. (2013). The garden of forking paths: Why multiple comparisons can be a problem, even when there is no "fishing expedition" or "p-hacking" and the research 
hypothesis was posited ahead of time. Technical report, Department of Statistics, Columbia University, New York, NY.

Goldin-Meadow, S. (2016). Why preregistration makes me nervous. APS Observer, 29(7).

Haak, L. L., Fenner, M., Paglione, L., Pentz, E., \& Ratner, H. (2012). ORCID: A system to uniquely identify researchers. Learned Publishing, 25(4), 259-264. https://doi.org/10.1087/20120404

Heng, Y. T., Wagner, D. T., Barnes, C. M., \& Guarana, C. L. (2018). Archival research: Expanding the methodological toolkit in social psychology. Journal of Experimental Social Psychology, 78(August 2017), 14-22. https://doi.org/10.1016/j.jesp.2018.04.012

John, L. K., Loewenstein, G., \& Prelec, D. (2012). Measuring the Prevalence of Questionable Research Practices With Incentives for Truth Telling. Psychological Science, 23(5), 524532. https://doi.org/10.1177/0956797611430953

Juster, F. T., \& Suzman, R. (1995). An Overview of the Health and Retirement Study. Journal of Human Resources, 30(Special Issue on the Health and Retirement Study: Data Quality and Early Results), 7-56.

Meltzoff, A., \& Moore, K. (1977). Imitation of facial and manual gestures by human neonates. Science, 12(1968), 75-79. https://doi.org/10.1126/science.198.4312.75

Meltzoff, A. N., Murray, L., Simpson, E., Heimann, M., Nagy, E., Nadel, J., ... Ferrari, P. F. (2017). Re-examination of Oostenbroek et al. (2016): evidence for neonatal imitation of tongue protrusion. Developmental Science, (July), e12609. https://doi.org/10.1111/desc.12609 
Nosek, B. A., Ebersole, C. R., DeHaven, A. C., \& Mellor, D. T. (2018). The preregistration revolution. Proceedings of the National Academy of Sciences, 2017(15), 201708274. https://doi.org/10.1073/pnas.1708274114

Nosek, B. A., \& Lindsay, D. S. (2018, March). Preregistration becoming the norm in psychological science. Observer.

Oostenbroek, J., Suddendorf, T., Nielsen, M., Redshaw, J., Kennedy-Costantini, S., Davis, J., ... Slaughter, V. (2016). Comprehensive longitudinal study challenges the existence of neonatal imitation in humans. Current Biology, 26(10), 1334-1338. https://doi.org/10.1016/j.cub.2016.03.047

Scott, S. (2013, July). Pre-registration would put science in chains. Times Higher Education.

Simon, D. A., Gordon, A. S., Steiger, L., \& Gilmore, R. O. (2015). Databrary: Enabling Sharing and Reuse of Research Video. Proceedings of the 15th ACM/IEEE-CE on Joint Conference on Digital Libraries, 279-280. https://doi.org/10.1145/2756406.2756951

Simonsohn, U., Nelson, L. D., \& Simmons, J. P. (2014). P-curve : A Key To The File Drawer. Journal of Experimental Psychology: General, 143(2), 534-547. https://doi.org/10.1037/a0033242

University of Michigan. 2018. Health and Retirement Study. Available at: http://hrsonline.isr.umich.edu/ (accessed June 22, 2018)

van 't Veer, A. E., \& Giner-Sorolla, R. (2016). Pre-registration in social psychology-A discussion and suggested template. Journal of Experimental Social Psychology, 67, 2-12. https://doi.org/10.1016/j.jesp.2016.03.004 
Wagenmakers, E. J., Wetzels, R., Borsboom, D., van der Maas, H. L. J., \& Kievit, R. A. (2012). An Agenda for Purely Confirmatory Research. Perspectives on Psychological Science, 7(6), 632-638. https://doi.org/10.1177/1745691612463078

Washburn, A. N., Hanson, B. E., Motyl, M., Skitka, L. J., Yantis, C., Wong, K. M., .. Carsel, T. S. (2018). Why do some psychology researchers resist adopting proposed reforms to research practices? A description of researchers' rationales. Advances in Methods and Practices in Psychological Science, 1(2), 166-173. https://doi.org/10.1177/2515245918757427

Watt, C. A., \& Kennedy, J. E. (2017). Options for prospective meta-analysis and introduction of registration-based prospective meta-analysis. Frontiers in Psychology, 7(JAN), 1-4. https://doi.org/10.3389/fpsyg.2016.02030

Weston, S.J., Ritchie, S.J., Rohrer, J.M., Przybylski, A.K. (2018). Recommendations for increasing the transparency of analysis of pre-existing datasets. Under review at Advanced Methods and Practices in Psychological Science, available at https://psyarxiv.com/zmt3q/

Whewell, W. (1858). Novum organon renovatum. JW Parker and Son.

Wicherts, J. M., Veldkamp, C. L. S., Augusteijn, H. E. M., Bakker, M., van Aert, R. C. M., \& van Assen, M. A. L. M. (2016). Degrees of freedom in planning, running, analyzing, and reporting psychological studies: A checklist to avoid p-hacking. Frontiers in Psychology, 7(NOV), 1-12. https://doi.org/10.3389/fpsyg.2016.01832 